\title{
Bioanalysis
}

\section{Microcontact printing in bioanalysis: where are we and where shall we be?}

\author{
"The success of microcontact printing of biomolecules in bioanalysis \\ lies in how much we can reduce the time between drying of 'inked' \\ stamp and printing of the biomolecules..."
}

First draft submitted: 22 June 2016; Accepted for publication: 23 August 2016; Published online: 9 September 2016

\section{Keywords: bioanalysis $\bullet$ fabrication $\bullet$ microcontact printing $\bullet$ PDMS}

Since the inception of microcontact printing $(\mu \mathrm{CP})$ in early 1990 s by Whitesides and coworkers [1,2] to control protein adsorption on cell culture surfaces, it has nowadays covered the widest picture and area of protein analysis research field. Basically, $\mu \mathrm{CP}$ is the transferring of the 'ink' pattern from the surface of a relief elastomeric stamp to a substrate through contact. The stamp is usually made of polydimethylsiloxane (PDMS) or its structured forms due to its low Young's modulus and high hydrophobicity (a contact angle of $\sim 110^{\circ}$ with water), while the 'ink' is usually made of molecules, such as alkanethiols or proteins/enzymes type biomolecules. Complete transfer of these biomolecules from the stamp to a substrate has been observed (almost 100\% efficiency) just after a few seconds of contact, without any loss of biological activity. In this way, $\mu \mathrm{CP}$ has simply fabricated functional patterns of proteins up to a scale where less than $0.1 \mathrm{k}$ molecules were placed in the sharp spots of the surface. Additionally, it has been suitable for fabrication of various biomolecules onto both the hydrophobic and hydrophilic substrates, such as various monolayers on gold, glass, poly(methylmethacrylate), silicon, silicon oxide or polystyrene. The reason behind the high resolution and contrast of the patterns fabricated by $\mu \mathrm{CP}$ of biomolecules, documented by atomic force microscopy, is the nondiffused behavior of adsorbed biomolecules' surfaces and the mechanical stability of the patterns of the stamp [3]. The success of $\mu \mathrm{CP}$ of biomolecules in bioanalysis lies in how much we can reduce the time between drying of 'inked' stamp and printing of the biomolecules because even 1 min delay may decrease the success of transfer substantially.

\section{Advanced present}

Over the last two decades and more, $\mu \mathrm{CP}$ has been developed as a high-throughput and precise technique for bioanalysis through several stages of development and research, which include the following:

- Being very hydrophobic in nature, PDMS was initially not found favorable for bioanalysis because when the 'ink' is composed of biomolecules that may lose their biological activity due to their hydrophilic nature. To sort out these issues, hydrophilicity of PDMS stamp surfaces has been enhanced by the following ways:

- Oxidation using UV/ozone or by oxygen plasma treatment methods;

- Coating various derivatives of polyethylene glycol;

- Plasma polarization methods.

All these methods have their own merits and demerits.

- In order to prevail over the shortcomings of PDMS, other polymers have also been proposed during the journey of bioanalysis based on $\mu \mathrm{CP}$; these include poly(ether-

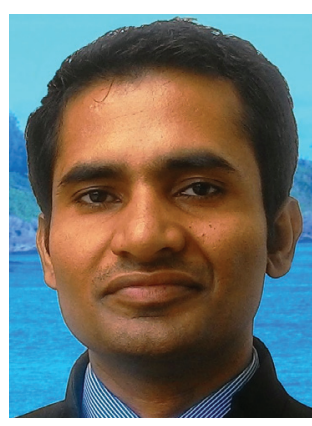

Rituraj Dubey

Department of Chemistry, Buddha Post Graduate College, Kushinagar - 274403, India and

Department of Chemistry, Indian Institute of Technology Roorkee, Roorkee - 247667, India

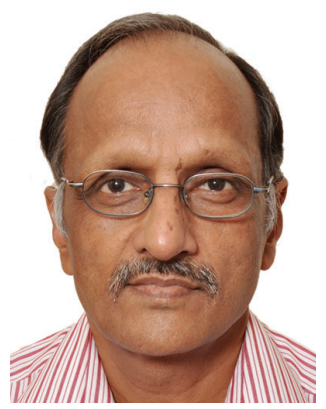

Ravi Bhushan

Author for correspondence:

Department of Chemistry, Indian Institute of Technology Roorkee, Roorkee - 247667, India

rbushfcy@iitr.ac.in

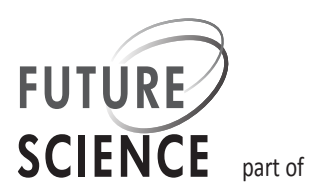


block-ester), hydrophilic thermoplastic elastomers [4], NOA 63-PEGDA polymer formed by UV curing of a urethane-based polymer, Norland Optical Adhesives 63 and poly(ethylene glycol) diacrylate [5], and a cross-linked hydrogel stamp [6]. These polymers are hydrophilic in nature and were found successful in one kind of bioanalysis, for example, protein analysis, as they reduced inking time and concentration even in aqueous environment without affecting the high precision of $\mu \mathrm{CP}$;

- The next challenge in the field was to microfabricate 'patterned' printing images of biomolecules. Besides, the high contrast of hydrophilic/hydrophobic nature of the printed and nonprinted regions appeared to be the crucial requirement. These patterned printing images were introduced in bioanalysis by incorporating the concept of self-assembled monolayers having different functionalities [7]. Thus, $\mu \mathrm{CP}$ was set to be the most appealing technique as it is capable of controlling the fabrication of biomolecules' thin films, and, hence, it has enhanced its importance in the field of bioanalysis by forecasting the generation of biomolecules' high-quality patterns on nonplanar substrates for biochips and other microfluidic-based bioanalysis applications;

\section{"The usage of microcontact printing lies in the fact that it is quite compatible with standard biomedical procedures, such as ELISA and fluorescence labeling."}

- Another interesting advancement we have seen in the past few years is patterning the same surface with several different biomolecules. Two approaches have been used for this purpose: sequential inking and printing, and parallel inking of a stamp followed by a single printing. Consequently, $\mu \mathrm{CP}$ has been found advantageous over standard arraying procedures of bioanalysis because only $\mu \mathrm{CP}$ can fabricate arbitrary patterns of different biomolecules on a single substrate by successive printing $[8]$;

- In order to offer a route to robust and high throughput, the fabrication of patterned surfaces was further extended up to nanometer level [9]. It is not easy to fabricate nanopattern of multiple moieties on small stamps but it becomes very useful when patterning over large area is required.

\section{References}

1 Prime K, Whitesides GM. Self-assembled organic monolayers: model systems for studying adsorption of proteins at surfaces. Science 252, 1164-1167 (1991).

\section{Promising future}

To date, $\mu \mathrm{CP}$ has become an indispensable tool for bioanalysis due to its simplicity, flexibility and efficiency in patterning of biomolecules, as well as its quite effective combination with microfluidic networks. It is capable of processing large bioactive surfaces in a single run containing arbitrary patterns. The usage of $\mu \mathrm{CP}$ lies in the fact that it is quite compatible with standard biomedical procedures, such as ELISA and fluorescence labeling.

Despite much advanced stage, in the present scenario, of bioanalytical application of $\mu \mathrm{CP}$, there are several sectors that need to be addressed and improved by research in future. For instance, improvement in the printing performance in terms of ease of operation and resolution, development of green printing and extension of application in several other fields of science are required to be explored. Although the contrast of the patterns fabricated by $\mu \mathrm{CP}$ can be extrapolated up to nanocontact printing by inventing new suitable materials for nanofabrication but the available methods for fabrication of nanostructured surfaces are not compatible with cheap and rapid processing for large-scale fabrication because they follow multistep syntheses; hence, a strategy for development of single-step fabricated cost-effective materials should be explored. Another important feature is to incorporate fatigue-resistant surfaces for robust printing so that the surface structure and functionality against external depreciation, mechanical beat and chemical adulteration may remain stable. We are predicting the future of $\mu \mathrm{CP}$ in developing superadvanced lab-on-a-chip devices as they usually require a small number of biomolecules for analysis, as well as their more essential role in digitalization of biochips and ecofriendly green printing with the reinforcement of science and the recognition of tenable reinforcement of $\mu \mathrm{CP}$ for bioanalysis purpose.

\section{Financial \& competing interests' disclosure}

The authors have no relevant affiliations or financial involvement with any organization or entity with a financial interest in or financial conflict with the subject matter or materials discussed in the manuscript. This includes employment, consultancies, honoraria, stock ownership or options, expert testimony, grants or patents received or pending, or royalties.

No writing assistance was utilized in the production of this manuscript.

2 Kumar A, Whitesides GM. Features of gold having micrometer to centimeter dimensions can be formed through a combination of stamping with an elastomeric stamp and an alkanethiol 'ink' followed by chemical etching. Appl. Phys. Lett. 63, 2002 (1993). 
3 Bernard A, Fitzli D, Sonderegger P et al. Affinity capture of proteins from solution and their dissociation by contact printing. Nat. Biotech. 19, 866-870 (2001).

4 Trimbach DC, Al-Hussein M, de Jeu WH, Decré M, Broer DJ, Bastiaansen CWM. Hydrophilic elastomers for microcontact printing of polar inks. Langmuir 20, 4738-4742 (2004).

5 Lee NY, Lim JR, Lee MJ, Park S, Kim SY. Multilayer transfer printing on microreservoir-patterned substrate employing hydrophilic composite mold for selective immobilization of biomolecules. Langmuir 22, 7689-7694 (2006).
6 Coq N, van Bommel T, Hikmet RA, Stapert HR, Dittmer WU. Self-supporting hydrogel stamps for the microcontact printing of proteins. Langmuir 23, 5154-5160 (2007).

7 Benor A, Hoppe A, Wagner V, Knipp D. Microcontact printing and selective surface dewetting for large electronic applications. Thin Solid Films 515, 7679-7682 (2007).

8 Lange SA, Benes V, Kern DP, Horber JKH, Bernard A. Microcontact printing of DNA molecules. Anal. Chem. 76, 1641-1647 (2004).

9 Ji S, Liu CC, Liu G, Nealey PF. Molecular transfer printing using block copolymers. ACS Nano 4, 599-609 (2010). 